\title{
Vertically Bounded Double Diffusive Convection in the Finger Regime: Comparing No-Slip versus Free-Slip Boundary Conditions
}

\author{
Yantao Yang, ${ }^{1, *}$ Roberto Verzicco, ${ }^{1,2}$ and Detlef Lohse ${ }^{1,3}$ \\ ${ }^{1}$ Physics of Fluids Group, MESA+ Research Institute, and J. M. Burgers Centre for Fluid Dynamics, \\ University of Twente, P.O. Box 217, 7500 AE Enschede, The Netherlands \\ ${ }^{2}$ Dipartimento di Ingegneria Industriale, University of Rome "Tor Vergata", Via del Politecnico 1, Roma 00133, Italy \\ ${ }^{3}$ Max-Planck Institute for Dynamics and Self-Organization, Am Fassberg 17, 37077 Göttingen, Germany \\ (Received 16 February 2016; revised manuscript received 18 September 2016; published 27 October 2016)
}

\begin{abstract}
Vertically bounded fingering double diffusive convection is numerically investigated, focusing on the influences of different velocity boundary conditions, i.e., the no-slip condition, which is inevitable in the lab-scale experimental researches, and the free-slip condition, which is an approximation for the interfaces in many natural environments, such as the oceans. For both boundary conditions the flow is dominated by fingers and the global responses follow the same scaling laws, with enhanced prefactors for the free-slip cases. Therefore, the laboratory experiments with the no-slip boundaries serve as a good model for the finger layers in the ocean. Moreover, in the free-slip case, although the tangential shear stress is eliminated at the boundaries, the local dissipation rate in the near-wall region may exceed the value found in the no-slip cases, which is caused by the stronger vertical motions of horizontally focused fingers and sheet structures near the free-slip boundaries. This counterintuitive result might be relevant for properly estimating and modeling the mixing and entrainment phenomena at free surfaces and interfaces widespread in oceans and geophysical flows.
\end{abstract}

DOI: 10.1103/PhysRevLett.117.184501

Double diffusive convection (DDC) - the convection flow where the fluid density is determined by two scalars with different molecular diffusivities-is ubiquitous in many natural environments. The terrestrial system of the greatest relevance is the ocean [1-4], where the density of seawater mainly depends on temperature and salinity. Originally proposed as an oceanographical curiosity [5], DDC has drawn lots of attention since it plays an important role in ocean mixing; e.g., see a comprehensive review in the recent book of Radko [6] and the references therein. A particularly fascinating phenomenon of DDC flows is the salt fingers, which occur when a fluid layer experiences an unstable salinity gradient and a stable temperature gradient. Salt fingers can even grow when the overall stratification is stable [7]. Conditions favoring salt fingers are present in most subtropic oceans [8]. Fingering DDC can induce intense vertical mixing [9], and may even attenuate the ocean signatures of climate changes [10].

Most early experiments on fingering convection employed a sharp interface from which the salt fingers grow and extend freely in the vertical direction [3]. When starting from a thick region with both temperature and salinity gradients, one finger layer or a stack of alternating convection and finger layers may develop, depending on the control parameters [11-13]. Three-dimensional direct numerical simulations (DNS) have provided detailed information on DDC, such as simulations in a fully periodic box with uniform background gradients [14-17] and those bounded by two parallel plates $[18,19]$. In the recent experiments using electrodeposition cells by Tilgner and co-workers [20,21], one single finger layer was observed between top and bottom boundaries for both stable and unstable stratification. Those experiments provide a good platform to investigate the vertical scalar transport and flow structures of finger layers. Our previous DNS successfully reproduced most key observations of the experiments, and good agreement was obtained between our numerical results and the experimental results [22-24].

However, an inevitable difference between the experiments and the ocean finger layers is that the experiments have been done with no-slip boundaries, which do not exist in the ocean. Therefore, the relevance of these experiments for ocean DDC flow has been questioned. To clarify the relevance of this difference, in the present Letter, by using DNS, we carry out a comparison between finger layers bounded by two no-slip plates, which are the same as the experiments (see, e.g., [20,21]), and those bounded by two free-slip plates, which model the ocean finger layers bounded by two homogeneous convection layers as in the thermohaline staircase. Similar studies were conducted for (rotating) Rayleigh-Bénard (RB) flow [25,26]. The current study is required if one wants to apply the experimental results of DDC flow to ocean flow [21]. Moreover, the comparison between different boundary conditions reveals some surprising characteristics of fingering DDC flow.

Consider DDC flow between two parallel plates that are perpendicular to the direction of gravity and separated by a 


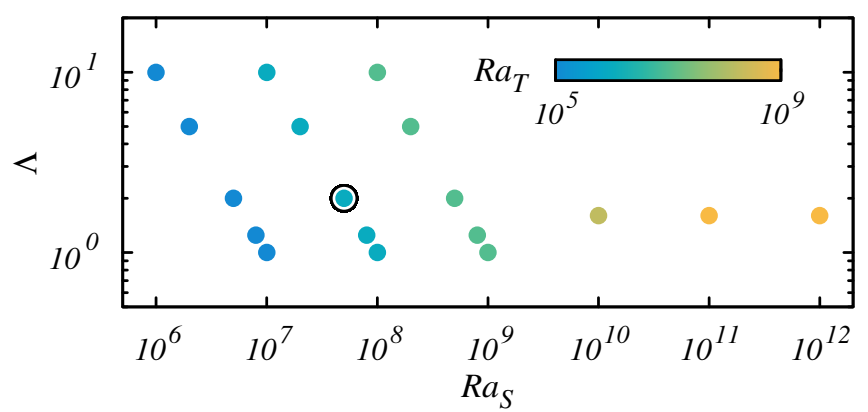

FIG. 1. The explored parameters shown in the $\mathrm{Ra}_{S}-\Lambda$ plane and colored by $\mathrm{Ra}_{T}$. The black circle marks the case shown in Fig. 2.

height $L$. At the two plates both temperature and salinity are kept constant. The two Prandtl numbers, i.e., the ratio between the kinematic viscosity $\nu$ and the scalar molecular diffusivity $\kappa$, are fixed at $\operatorname{Pr}_{T}=7$ and $\operatorname{Pr}_{S}=700$, which are the typical values of subtropic seawater. We confine ourselves in the finger regime, namely, the top plate has both higher salinity and temperature. The flow is driven by the salinity difference $\Delta_{S}$ between two plates and stabilized by the temperature difference $\Delta_{T}$. The strength of the buoyancy force associated with the scalar field $\zeta=T$ or $S$ is measured by the Rayleigh number $\mathrm{Ra}_{\zeta}=$ $\left(g \beta_{\zeta} L^{3} \Delta_{\zeta}\right) /\left(\kappa_{\zeta} \nu\right)$, with $g$ being the gravitational acceleration and $\beta_{\zeta}$ the positive expansion coefficient, respectively. The density ratio, which reflects the relative strength of the buoyancy force induced by the temperature difference to that by the salinity difference, can then be calculated as $\Lambda=\left(\beta_{T} \Delta_{T}\right) /\left(\beta_{S} \Delta_{S}\right)=\left(\operatorname{Pr}_{S} \operatorname{Ra}_{T}\right) /\left(\operatorname{Pr}_{T} \operatorname{Ra}_{S}\right)$.

The flow quantities are nondimensionalized by $L, \Delta_{T}$, $\Delta_{S}$, and the free fall velocity $U=\sqrt{g \beta_{S} \Delta_{S} L}$. We numerically solve the incompressible Navier-Stokes equation within the Oberbeck-Boussinesq approximation for the velocity $u_{i}$, with $i=1,2,3$, pressure $p$, and nondimensionalized temperature $\theta$ and salinity $s$, respectively. The subscript " 3 " denotes the component in the vertical direction opposite to the gravity. The details of the numerical work can be found in Refs. [27,28]. At the two plates either no-slip or free-slip boundary conditions are imposed for the tangential velocity components and the nonpenetration condition for the normal velocity component, respectively. In the horizontal directions we apply periodic boundary conditions. The aspect ratio is chosen such that the horizontal size of the domain is much larger than the horizontal length scale of the salt fingers.

The explored phase space of our numerical simulations is shown in Fig. 1 . The salinity Rayleigh number $\mathrm{Ra}_{S}$ ranges from $10^{6}$ to $10^{12}$ with $1 \leq \Lambda \leq 10$, i.e., in the salt finger regime. For every pair of $\left(\mathrm{Ra}_{S}, \Lambda\right)$ two simulations were conducted with either no-slip or free-slip boundary condition. Initially, the fluid is at rest, the temperature field has a vertically linear distribution, and the salinity field is uniform and equal to the mean of the values at the two plates, respectively. These initial fields are the same as those in the experiments [20,21]. Figures 2(a) and 2(b) display the threedimensional volume rendering of salt fingers for $\left(\mathrm{Ra}_{S}, \Lambda\right)=$ $\left(5 \times 10^{7}, 2.0\right)$ with different boundary conditions. The color map and opacity settings are exactly the same in the two plots. The flow morphology is essentially the same for the two boundary conditions: The vertically oriented salt fingers occupy the whole bulk region, while near the plates sheetlike structures connect the roots of the adjacent fingers. Usually fingers are associated with slender convection cells. Compared to the no-slip cases, the salt fingers in the freeslip case are much stronger; i.e., the fluid moves with larger vertical velocity and high salinity anomaly occupies more space in the bulk region. This is expected since the no-slip boundaries not only exert the vertically geometric confinement as the free-slip boundaries do, but they also require the horizontal velocity to be zero at the two plates. Thus, in the no-slip case the recirculation near the boundary within each convection cell is weakened, and the salt fingers are not as strong as in the free-slip case.

The three most important responses of the system are the dimensionless transfer rates for the two scalars $\mathrm{Nu}_{S}=\left(\left\langle u_{3} s\right\rangle-\kappa_{S}\left\langle\partial_{3} s\right\rangle\right) /\left(\kappa_{S} \Delta_{S} L^{-1}\right)$ and $\mathrm{Nu}_{T}=\left(\left\langle u_{3} \theta\right\rangle-\right.$ $\left.\kappa_{T}\left\langle\partial_{3} \theta\right\rangle\right) /\left(\kappa_{T} \Delta_{T} L^{-1}\right)$, and the dimensionless flow velocity $\operatorname{Re}=U_{\mathrm{rms}} L / \nu$. Here, $\langle\cdot\rangle$ denotes the average over the entire domain and time, and $U_{\text {rms }}$ is the rms value of the velocity magnitude. The dependences of the three

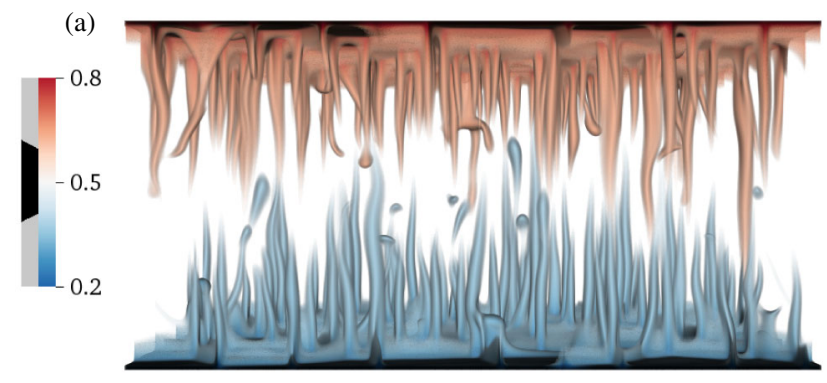

(b)

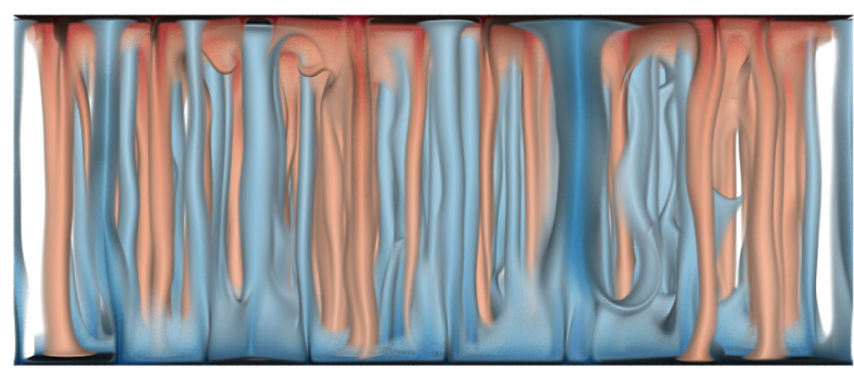

FIG. 2. The volume rendering of salt fingers for $\left(\mathrm{Ra}_{S}, \Lambda\right)=\left(5 \times 10^{7}, 2.0\right)$ (marked by a black circle in Fig. 1) with (a) the no-slip boundary condition and aspect ratio 2.0 and (b) the free-slip boundary condition and aspect ratio 2.4 , respectively. The two plots share the same color map and opacity settings. 


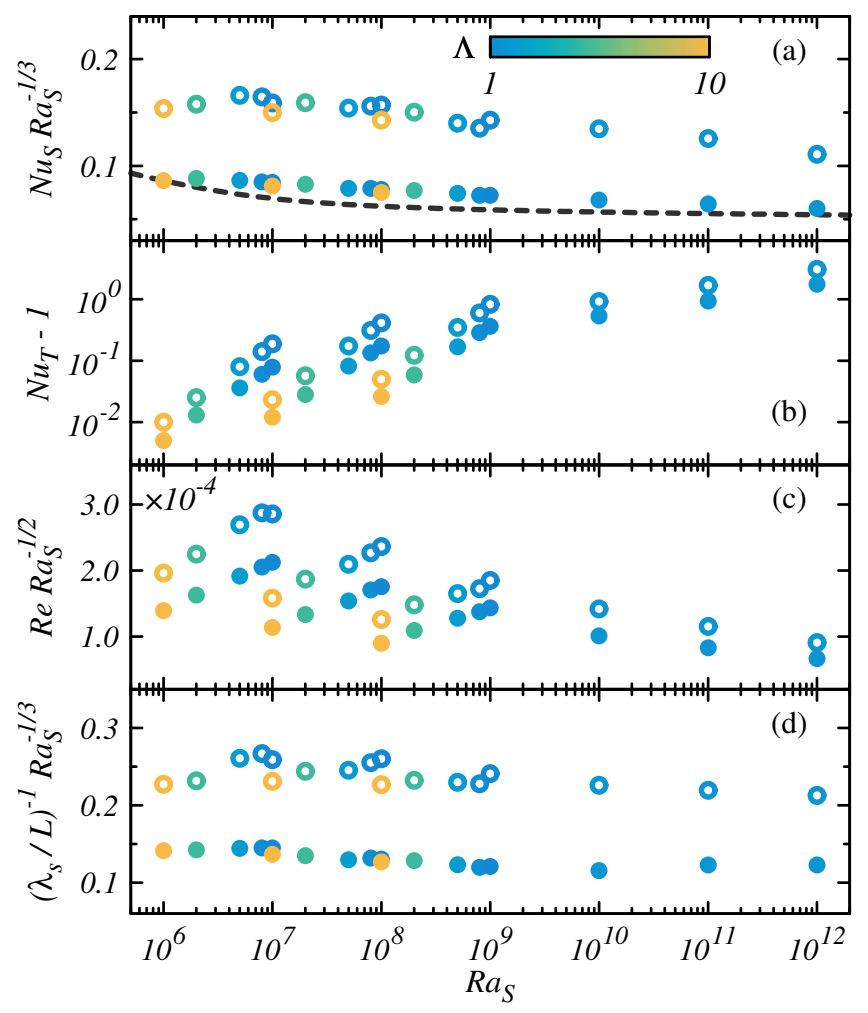

FIG. 3. System responses versus $\mathrm{Ra}_{S}$. (a) $\mathrm{Nu}_{S}$ compensated by $\mathrm{Ra}_{S}^{1 / 3}$, (b) $\mathrm{Nu}_{T}-1$, (c) $\mathrm{Re}$ compensated by $\mathrm{Ra}_{S}^{1 / 2}$, and (d) $\left(\lambda_{s} / L\right)^{-1}$ compensated by $\mathrm{Ra}_{S}^{1 / 3}$. The free-slip cases are marked by open symbols and the no-slip cases by solid symbols, respectively. Symbols are colored according to the density ratio $\Lambda$. The dashed line in (a) represents the Grossmann-Lohse prediction.

quantities as functions of $\mathrm{Ra}_{S}$ are plotted in Figs. 3(a)-3(c). Since $\mathrm{Nu}_{T}$ is very close to the conductive value 1 , we plot in Fig. 3(b) only the convective flux $\mathrm{Nu}_{T}-1$. The effects of different boundary conditions revealed by Fig. 3(a)-3(c) are consistent with the flow fields shown in Fig. 2 in two aspects. First, all three quantities are enhanced by replacing the no-slip boundary condition with the free-slip one, which is attributed to stronger salt fingers, as shown in Fig. 2(b) as compared to Fig. 2(a). The salt fingers in the free-slip cases move faster in the vertical direction and, therefore, the scalar transfer is more efficient. Second, the quantities follow very similar scaling laws for different boundary conditions, which reflects the fact that the flow morphology is essentially the same, i.e., dominated by salt fingers. In Fig. 3(a) we also compare $\mathrm{Nu}_{S}$ with the Grossmann-Lohse theory [29-33] for the no-slip cases. The agreement is very good, which has also been found in our previous studies [22-24]. Our previous studies with no-slip boundary conditions revealed that for fixed $\mathrm{Ra}_{S}$, as $\Lambda$ increases within the finger regime, $\mathrm{Nu}_{S}$ only changes slightly and Re decreases significantly [22,24]. The present results suggest that it is also true for the free-slip cases; see Figs. 3(a) and 3(c).
Compared to the no-slip cases, the thickness $\lambda_{s}$ of the salinity boundary layer is smaller in the free-slip cases. Here $\lambda_{s}$ is defined as the distance between the first peak of the $s_{\text {rms }}$ profile and the adjacent boundary. Our results indicate that for both boundary conditions, $\lambda_{s}$ scales as $\mathrm{Nu}_{S}^{-1}$. In Fig. 3(d) we plot the reciprocal value $\left(\lambda_{s} / L\right)^{-1}$ compensated by $\mathrm{Ra}_{S}^{-1 / 3}$, which follows a very similar trend as $\mathrm{Nu}_{S}$ shown in Fig. 3(a).

The above observations indicate that salt fingers are robust with respect to different velocity boundary conditions, and, therefore, the exponents of the scaling laws for $\mathrm{Nu}_{S}, \mathrm{Nu}_{T}$, and $\mathrm{Re}$ are also the same. According to the global balance between the Nusselt numbers and the total dissipation $[22,28]$, the global dissipation rate must be higher for the free-slip cases than that for the no-slip cases. In Fig. 4(a) we plot the horizontally averaged mean profiles of $D_{a}=\left\langle S_{i j} S_{i j}\right\rangle_{h}$ calculated from the flow fields shown in Fig. 2. Here, $S_{i j}=\left(\partial_{j} u_{i}+\partial_{i} u_{j}\right) / 2$ is the strain-rate tensor and it relates to the local dissipation rate by $\varepsilon=2 \nu S_{i j} S_{i j}$. Indeed, the dissipation rate of the free-slip case is higher in the bulk region than that of the no-slip case. Meanwhile, near the boundary the dissipation rate is also enhanced in the free-slip case. This is surprising since by imposing the free-slip boundary condition we eliminate the shear stress at the boundary. The increase in the dissipation rate near the
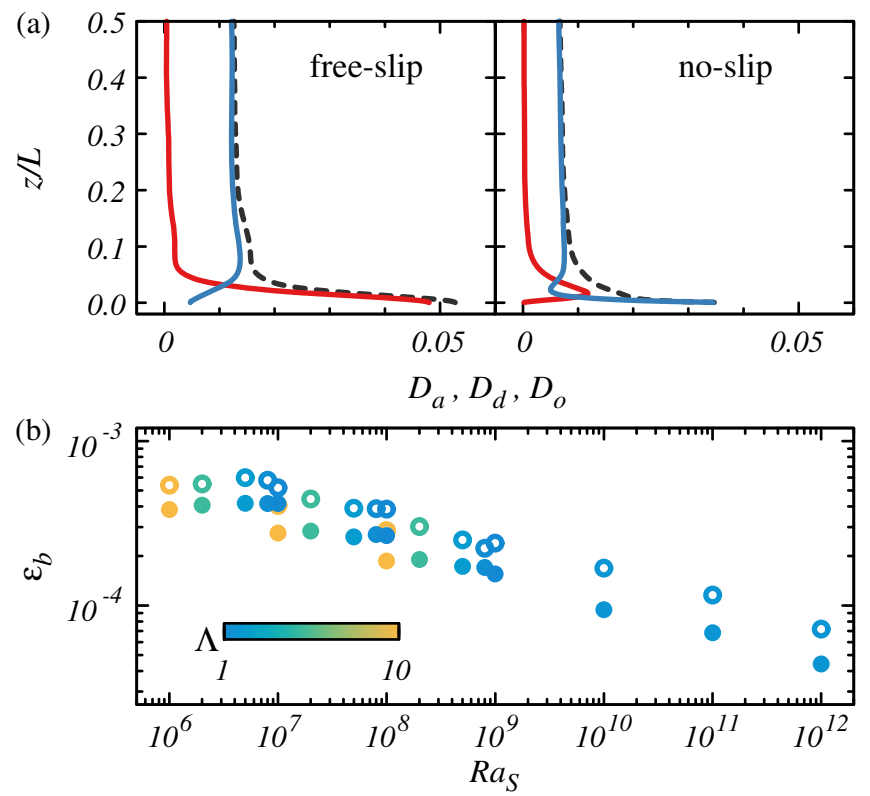

FIG. 4. (a) The mean profiles of $D_{a}=\left\langle S_{i j} S_{i j}\right\rangle_{h}$ (black dashed lines) and the contributions from the diagonal components of the strain-rate tensor $D_{d}=\left\langle\Sigma_{i=j}\left(S_{i j} S_{i j}\right)\right\rangle_{h}$ (red lines) and the offdiagonal components $D_{o}=\left\langle\Sigma_{i \neq j}\left(S_{i j} S_{i j}\right)\right\rangle_{h}$ (blue lines) for the free-slip case and no-slip cases. The control parameters are $\mathrm{Ra}_{S}=$ $5 \times 10^{7}$ and $\Lambda=2.0$. Because of the symmetry about $z=0.5$, only the lower half of the domain is shown here. (b) The averaged dissipation rate $\varepsilon_{b}$ at boundary. The symbols and color map are the same as in Fig. 3. 
(a) $u_{3}$

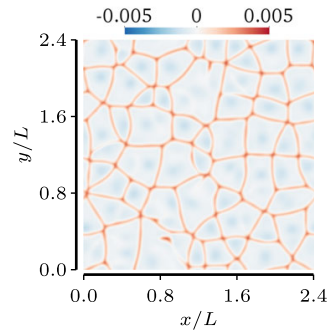

(b) $s$

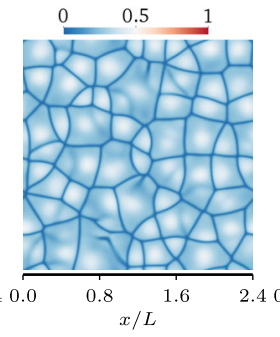

(c) $D_{d}$

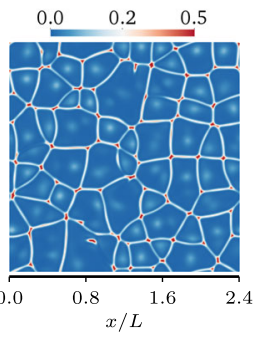

FIG. 5. The contours on a horizontal plane near the bottom plate at $z / L=0.005$ for the flow field shown in Fig. 2(b) with the free-slip boundary condition. (a) The vertical velocity $u_{3}$, (b) the salinity $s$, and (c) $D_{d} \equiv \Sigma_{i=j}\left(S_{i j} S_{i j}\right)$, which dominates the local dissipation rate.

boundaries when the no-slip boundary condition is replaced by the free-slip one is consistently observed for all Rayleigh numbers we simulated, as shown in Fig. 4(b). Previous studies reported similar behaviors in the RB flow with no-slip or free-slip boundary conditions [25].

To clarify the origin of the high dissipation rate, we divide $S_{i j} S_{i j}$ into the contribution from the diagonal components of $S_{i j}$, i.e., $D_{d} \equiv\left\langle\Sigma_{i=j} S_{i j} S_{i j}\right\rangle_{h}$, and that from the off-diagonal components, $D_{o} \equiv\left\langle\Sigma_{i \neq j}\left(S_{i j} S_{i j}\right)\right\rangle_{h}$. The mean profiles of the two parts are also plotted in Fig. 4(a). In the bulk region, the dissipation for both boundary conditions is dominated by the off-diagonal components $D_{o}$. Further examination of the data reveals that the largest contribution is from the horizontal gradient of the vertical velocity, which corresponds to the shear among salt fingers as they move in the vertical direction. The free-slip case allows stronger fingers with larger vertical velocity; thus, the dissipation rate is also higher than that for the no-slip case at the same control parameters.

Near the boundary the situation is totally different. For the free-slip case the dissipation near the boundaries is dominated by $D_{d}$, as shown in Fig. 4(a) for $\mathrm{Ra}_{S}=5 \times 10^{7}$ and $\Lambda=2.0$. This strong dissipation can be directly connected to the flow structures near two plates. In Fig. 5 we show, for the free-slip case with the same parameters, the contours of vertical velocity $u_{3}$, salinity $s$, and the dominant terms of dissipation rate $D_{d}$ on a horizontal plane $z / L=0.005$ which is very close to the bottom plate. At this height the local dissipation is dominated by tangential shear stress in the no-slip case. The sheet structures are very distinct in the contours of both $u_{3}$ and $s$. These sheet structures rise from the bottom plate and carry a large salinity anomaly. The ascending fingers usually grow from the intersections of the sheet structures. When these structures move upward, they induce strong converging flows in the horizontal directions and, therefore, large dissipation occurs as shown in Fig. 5(c). Note that the locations with high dissipation rates coincide with the sheet structures observed in Figs. 5(a) and 5(b). Meanwhile, the descending fingers from the top plates decelerate as they reach the bottom plate and drive the expanding flow in the horizontal directions within the convection cells separated by the sheet structures, which correspond to the large negative $u_{3}$ inside the cells in Fig. 5(a) and high salinity at the same locations in Fig. 5(b). Figure 5(c) indicates that at these locations the dissipation rate is also large.

At the no-slip boundary the velocity must be zero. In the thin layer adjacent to the plate, as shown in Fig. 4(a), the dissipation mainly originates from $D_{o}$ and the contribution from the diagonal components decreases to zero on the boundary. This is similar to a boundary layer where the vertical gradient of the horizontal velocity is the major source of dissipation. Above this quasiboundary layer region, however, there exists a region where $D_{d}$ has a peak and it is larger than $D_{o}$. This is where the sheet structures and fingers start to grow and the mechanism of large dissipation is essentially the same as the high dissipation rate near the free-slip boundary.

In conclusion, our results suggest that the vertically bounded fingering DDC is insensitive to different velocity boundary conditions, i.e., the no-slip or free-slip types, in the sense that for different boundary conditions (i) the flow morphology is basically the same and (ii) the Nusselt numbers of the two scalars and the Reynolds number exhibit similar dependences on $\mathrm{Ra}_{s}$. The free-slip boundary conditions allow stronger salt fingers, which results in higher scalar transfers and flow velocity. Despite the fact that the tangential shear stress is eliminated at the free-slip boundaries, in a thin layer adjacent to the boundaries the local dissipation rate can be higher than that for the no-slip cases. The large dissipation near the free-slip boundaries is dominated by the contributions from the diagonal components of the strain rate tensor, which are associated to the converging or expanding flows in the horizontal directions induced by the vertical motions of the salt fingers.

Two important indications can be obtained from the current study. First, the experiments for the DDC flows bounded by no-slip walls can still provide useful information, especially for the scaling laws and salt fingers in the bulk region. Only the prefactors should be reevaluated for ocean salt-finger layers. Second, the high dissipation rate near the free-slip boundaries implies that large dissipation is also likely to exist at interior interfaces, such as the boundaries of ocean salt-finger layers.

This study is supported by Dutch Foundation for Fundamental Research on Matter (Stichting FOM), and by the Netherlands Center for Multiscale Catalytic Energy Conversion (MCEC), an NWO Gravitation program funded by the Ministry of Education, Culture and Science of the government of the Netherlands. The computing resource was provided by the Dutch national e-infrastructure of SURFsara and the FERMI supercomputer based in CINECA, Italy through the PRACE Project No. 2015133124. 
*Corresponding author. yantao.yang@utwente.nl

[1] J. Turner, Annu. Rev. Fluid Mech. 17, 11 (1985).

[2] R. W. Schmitt, Annu. Rev. Fluid Mech. 26, 255 (1994).

[3] R. W. Schmitt, Progr. Oceanogr. 56, 419 (2003).

[4] R. W. Schmitt, J. R. Ledwell, E. T. Montgomery, K. L. Polzin, and J. M. Toole, Science 308, 685 (2005).

[5] H. Stommel, A. B. Arons, and D. Blanchard, Deep Sea Res. 3, 152 (1956).

[6] T. Radko, Double-Diffusive Convection (Cambridge University Press, Cambridge, United Kingdom, 2013).

[7] M. E. Stern, Tellus 12, 172 (1960).

[8] Y. You, Deep Sea Res. 49, 2075 (2002).

[9] C. Lee, K. Chang, J. Lee, and K. J. Richards, Geophys. Res. Lett. 41, 7964 (2014).

[10] G. C. Johnson and K. A. Kearney, Geophys. Res. Lett. 36, L21603 (2009).

[11] P. F. Linden, J. Geophys. Res. Oceans 83, 2902 (1978).

[12] R. Krishnamurti, J. Fluid Mech. 483, 287 (2003).

[13] R. Krishnamurti, J. Fluid Mech. 638, 491 (2009).

[14] E. Rosenblum, P. Garaud, A. Traxler, and S. Stellmach, Astrophys. J. 731, 66 (2011).

[15] S. Stellmach, A. Traxler, P. Garaud, N. Brummell, and T. Radko, J. Fluid Mech. 677, 554 (2011).

[16] A. Traxler, S. Stellmach, P. Garaud, T. Radko, and N. Brummell, J. Fluid Mech. 677, 530 (2011).

[17] T. Radko, J. Flanagan, S. Stellmach, and M.-L. Timmermans, J. Phys. Oceanogr. 44, 1285 (2014).

[18] T. Radko and M. E. Stern, J. Fluid Mech. 425, 133 (2000).
[19] F. Paparella and J. von Hardenberg, Phys. Rev. Lett. 109, 014502 (2012).

[20] E. Hage and A. Tilgner, Phys. Fluids 22, 076603 (2010).

[21] M. Kellner and A. Tilgner, Phys. Fluids 26, 094103 (2014).

[22] Y. Yang, E. P. van der Poel, R. Ostilla-Mónico, C. Sun, R. Verzicco, S. Grossmann, and D. Lohse, J. Fluid Mech. 768, 476 (2015).

[23] Y. Yang, R. Verzicco, and D. Lohse, Proc. Natl. Acad. Sci. U.S.A. 113, 69 (2016).

[24] Y. Yang, R. Verzicco, and D. Lohse, J. Fluid Mech. 802, 667 (2016).

[25] K. Petschel, S. Stellmach, M. Wilczek, J. Lülff, and U. Hansen, Phys. Rev. Lett. 110, 114502 (2013).

[26] R. Kunnen, R. Ostilla-Mónico, E. van der Poel, R. Verzicco, and D. Lohse, J. Fluid Mech. 799, 413 (2016).

[27] R. Ostilla-Mónico, Y. Yang, E. P. van der Poel, D. Lohse, and R. Verzicco, J. Comput. Phys. 301, 308 (2015).

[28] See Supplemental Material at http://link.aps.org/ supplemental/10.1103/PhysRevLett.117.184501 for details of numerical simulations.

[29] S. Grossmann and D. Lohse, J. Fluid Mech. 407, 27 (2000).

[30] S. Grossmann and D. Lohse, Phys. Rev. Lett. 86, 3316 (2001).

[31] S. Grossmann and D. Lohse, Phys. Rev. E 66, 016305 (2002).

[32] S. Grossmann and D. Lohse, Phys. Fluids 16, 4462 (2004).

[33] R. J. A. M. Stevens, E. P. van der Poel, S. Grossmann, and D. Lohse, J. Fluid Mech. 730, 295 (2013). 